\title{
Fast-tracking morphogen diffusion
}

\author{
Olivier Cinquin ${ }^{1,2,3}$ \\ 1: CoMPLEX, UCL \\ 2:Department of Anatomy and Developmental Biology \\ University College London \\ Gower Street, London WC1E 6BT, U.K. \\ 3:Laboratoire TIMC-IMAG-CNRS UMR 5525, Université Joseph Fourier, Grenoble 1 \\ Faculté de Médecine, Domaine de la Merci, 38706 La Tronche, France \\ Correspondence: o.cinquin@ucl.ac.uk \\ FAX: +44 2073835519
}

Journal of Theoretical Biology 238(3), pp532-540 (2006)

\section{Abstract}

The readout of morphogen concentrations has been proposed to be an essential mechanism allowing embryos to specify cell identities (Wolpert Trends Genet 12 (1996) 359), but theoretical and experimental results have led to conflicting ideas as to how useful concentration gradients can be established. In particular, it has been pointed out that some models of passive extracellular diffusion exhibit traveling waves of receptor saturation, inadequate for the establishment of positional information. Two alternative (but not mutually exclusive) models are proposed here, which are based on recent experimental results highlighting the roles of extracellular glycoproteins and morphogen oligomerization. In the first model, inspired from the interactions of Dally and Dally-like with Wingless and Decapentaplegic in the third-instar Drosophila wing disc, two morphogen populations are considered: one in a cell-membrane phase, and another one in an extracellular-matrix phase, which does not interact with receptors; in the second model, inspired from biochemical studies of Sonic Hedgehog, morphogen oligomers are considered to diffuse freely without interacting with receptors. The existence of a dynamic sub-population of freely-diffusing morphogen allows the system to establish a gradient of bound receptor, which is suitable for the specification 
of positional information. Recent experimental results are discussed within the framework of these models, as well as further possible experiments. The role of Notum in the setup of the Wingless gradient is also shown to be likely not to involve a gradient in Notum distribution, even though Notum is only expressed close to the source of Wingless synthesis.

Abbreviations: Dll: Dally; Dlp: Dally-like; Dpp: Decapentaplegic; Wg: Wingless; Shh: Sonic Hedgehog

\section{Introduction}

The third instar Drosophila wing disc has provided examples of molecules forming morphogen gradients (reviewed by Cadigan, 2002). Whether the transport of morphogens relies on passive extracellular diffusion has been a source of intense debate (see for example Kerszberg and Wolpert, 1998, Lander et al., 2002, Kruse et al., 2004), but recent experimental data has dealt a new hand by greatly clarifying the role of extracellular proteoglycans in the establishment of morphogen gradients (Baeg et al., 2004, Belenkaya et al., 2004, Han et al., 2004, 2005); mathematical models are yet to be developed to take this role into account.

It was first observed by Kerszberg and Wolpert (1998), and confirmed by Lander et al. (2002), that a simple system of morphogen diffusion and receptor binding will generally create a traveling wave of receptor saturation and not a stable gradient of bound receptor. It has been shown that this problem can be alleviated by hypothesizing a mechanism of ligand-receptor complex degradation and slow association constants (Lander et al., 2002), the possibility of signaling for internalized complexes (Lander et al., 2002), similar to the ligand-triggered, but ligand-free, receptor dimers of Kerszberg and Wolpert (1998), or self-enhanced ligand degradation (Eldar et al., 2003). We propose here two other simple mechanisms for suitably-shaped morphogen gradients to arise, one based on morphogen oligomerization, and one based on the role of extracellular glycoproteins. The latter model illustrates what the role of extracellular glycoproteins could be, and also provides a specific insight into the establishment of the Wingless $(\mathrm{Wg})$ gradient, one of the morphogens identified in the Drosophila wing disc.

\subsection{Glycoprotein-mediated phase repartition}

This model is inspired from the Wg (Zecca et al., 1996) and Decapentaplegic (Dpp; Nellen et al., 1996) morphogen gradients in the Drosophila wing-disc, but could also apply to other gradients, such as the Sonic Hedgehog (Shh) 
gradient in the developing neural tube (Briscoe et al., 2001). Dally (Tsuda et al., 1999; abbreviated Dll) and Dally-like (Khare and Baumgartner, 2000, Baeg et al., 2001; abbreviated Dlp) are cell-surface heparan sulfate proteoglycans, tethered to the membrane by a GPI anchor (ie, glypicans), which have been shown to influence both Dpp and Wg signalling. In the Drosophila wing disc, Wg and Dpp cannot diffuse into clones deficient for both Dll and Dlp, or into clones deficient for heparan-sulfate synthesis (Belenkaya et al., 2004, Han et al., 2005); it has been proposed that Dll acts as a Wg co-receptor, while Dlp mediates Wg movement across cells (Baeg et al., 2001), but Dll seems to also have a role in mediating inter-cellular movement (Han et al., 2005). Importantly, Dlp can be released into the extracellular matrix by the secreted protein Notum (Kreuger et al., 2004), and Dll-Notum interactions have been shown to be essential for wing-disc patterning (Han et al., 2005), suggesting that Dll could also be released by Notum. Notum is mainly expressed in the region of high Wg signalling, and can itself be released into the extracellular matrix (Gerlitz and Basler, 2002, Giráldez et al., 2002). Notum would not be taken into account in a model for Dpp gradient establishment (see below for a further discussion of Notum as regards the Wg and Dpp gradients).

In the model proposed here, and illustrated in Figure 1 Dll and Dlp are considered as a single entity (noted as $d$ in the equations detailed in Appendix A.4), which binds to the morphogen $m$. Two populations are considered, one in the extracellular matrix (EM), and one on the cell membrane (mem); cell membrane-attached $d$ molecules are relased into the extracellular matrix by Notum $(n)$. The crucial hypothesis of the model is that the morphogen equilibrates between the extracellular matrix and membrane phases, following the repartition of $d$. Extracellular morphogen is injected at the left boundary, and Notum synthesized (and considered to be immediately secreted) over the first $10 \mu \mathrm{m}$. Both free and bound receptor populations are taken into account, with a fixed rate of receptor synthesis, and unregulated degradation.

In order for the model to be compatible with the experimental data showing the absence of diffusion of morphogen into clones deficient for Dll and Dlp, only the EM morphogen and Notum are considered to diffuse.

\subsection{Shh-like oligomerization}

Shh has been shown to oligomerize, and it has been proposed that the higherorder multimers mediate long range signalling (Zeng et al., 2001, Chen et al., 2004, Feng et al., 2004). The relative signaling potencies of the different forms have been debated, but it seems reasonable that there should be a dynamic equilibrium between them (even if no interconversion was observed 


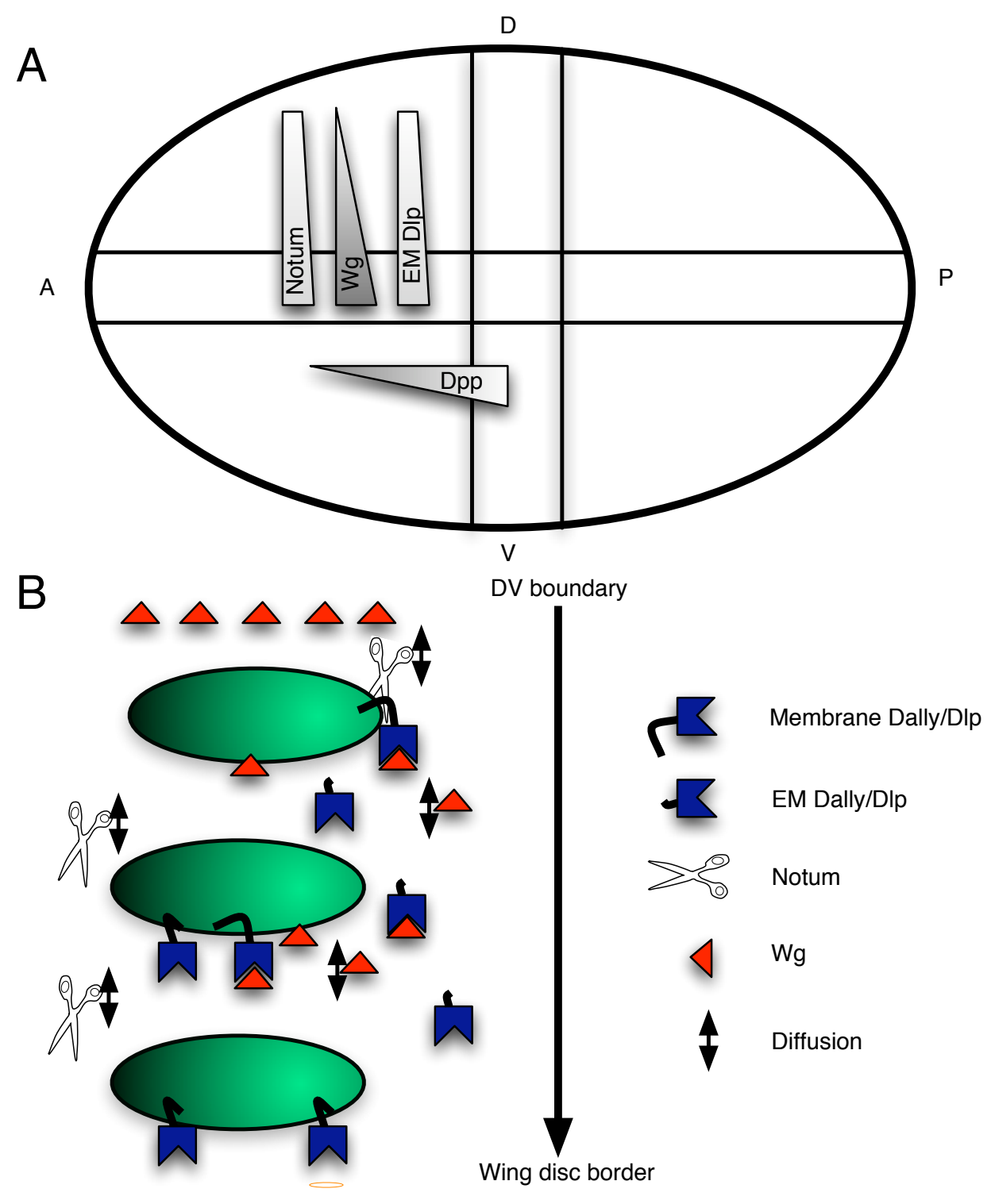

Figure 1: (A) Distribution of Wg, Dpp, Dll/Dlp, and Notum in a Drosophila third instar wing disc. (B) Glycoprotein phase-repartition model for $\mathrm{Wg}$ signaling. 
in vitro by Chen et al., 2004), and that signaling could be mediated by the monomeric form, while multimeric forms diffuse without interacting with the receptors. The available biochemical data is not sufficient to propose one specific Shh multimerization scheme; different oligomerization extents have been reported, from 6-mers to about 30-mers. Therefore, in order to assess the possibility for such multimerization to provide a suitable gradient of receptor-bound monomer, an arbitrary, generic scheme was adopted, in which any two oligomers can combine to create one of higher size, provided that the size of the product is smaller than the maximum being considered. The reactions are reversible, and any oligomer can split into two smaller products. Equations are detailed in section A.5.

\section{Results}

Simulations were run with successive sets of parameters sampled at random from the ranges detailed in Table 1. A set of parameters was considered to be suitable if the following conditions on the gradient of morphogen-bound receptor were met:

- about 3 hours after the start of the simulation, the concentration of morphogen-bound receptor was sufficiently close to linearity (as per a measure described in the appendix)

- after a further 3 hours of simulation, the gradient stayed with $30 \%$ of its original values

- the range of the gradient was greater than 2-fold

- the concentration of bound-receptor at the high end was greater than 30nM (corresponding to 100 bound receptor molecules, following the calculations in Lander et al., 2002), and more than $2 \%$ of the receptors were bound to morphogen $75 \mu \mathrm{m}$ into the field.

\subsection{Glycoprotein phase-repartition with localized No- tum synthesis}

Out of more than 100,000 parameter sets tested, about $0.5 \%$ met the conditions above, with gradient ranges of up to 34-fold (range average was 4). Remarkably, satisfactory parameter sets spanned the entire sampling range for each individual parameter, even though the ranges were chosen to be very wide, showing that the structure of the system can accommodate a 


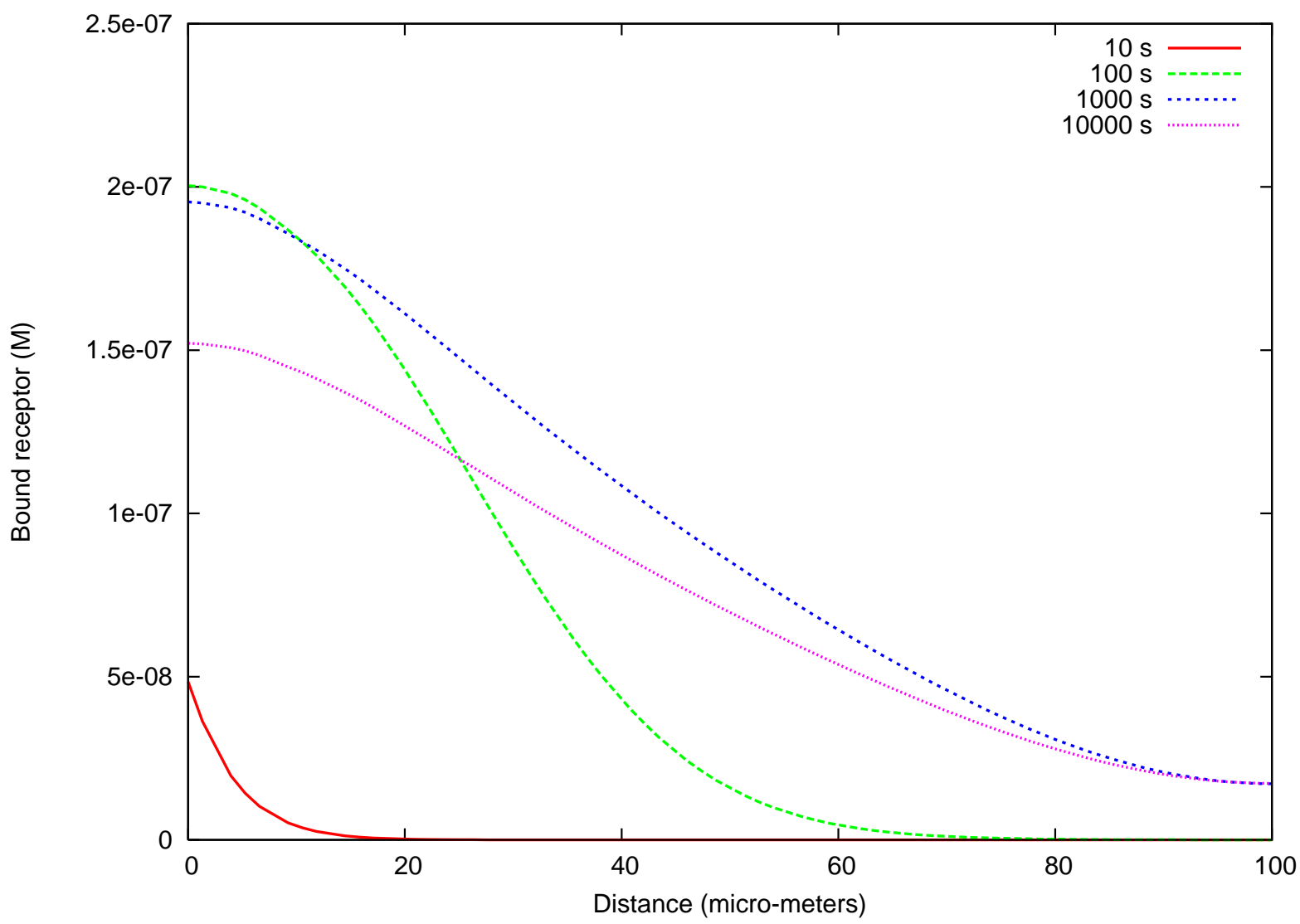

Figure 2: Example of a gradient of bound receptor which meets the criteria described in section 3.1 10000s after the start of the simulation. 


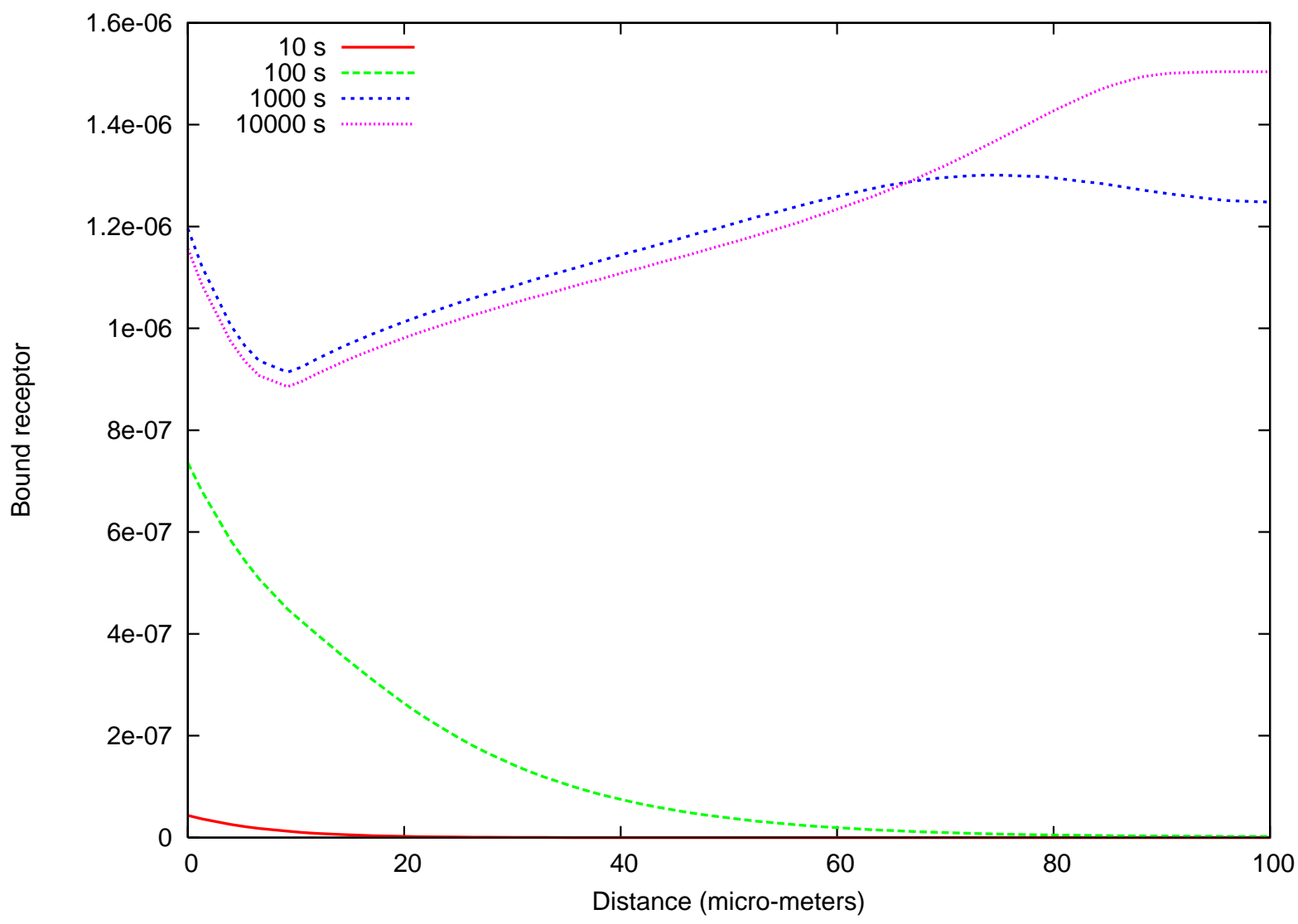

Figure 3: Example of a non-monotonous gradient of bound receptor. 


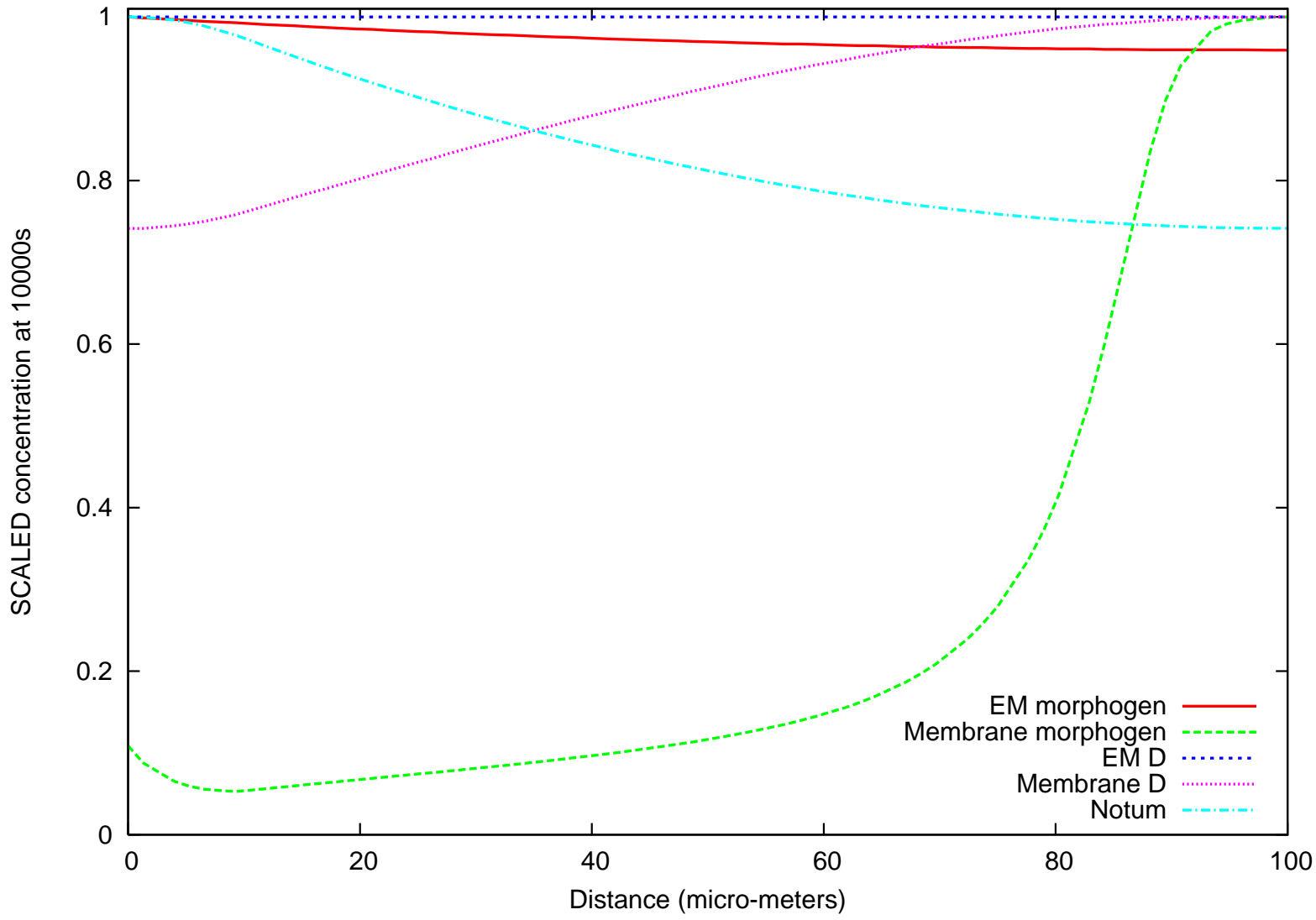

Figure 4: Concentrations of other elements of the system, for the same parameter values as in Figure 3, at 10000s. The curves were scaled with their highest value so their variations would be visible on the same graph. 


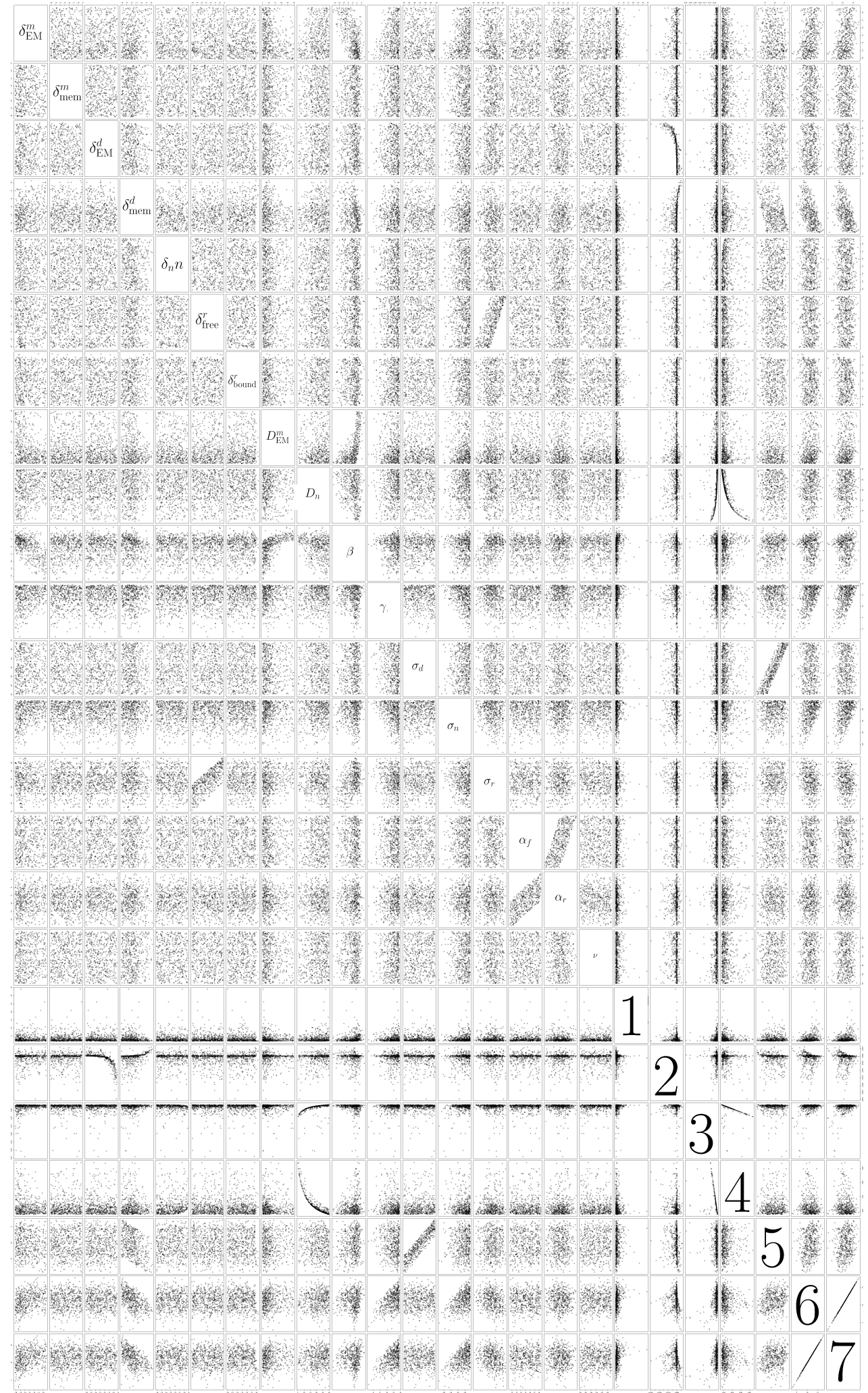

Figure 5: Pairwise plot of parameters for which the model with localized Notum synthesis gives rise to a gradient of bound receptor meeting the conditions set out in section 3.1. 1: $\frac{r_{\text {bound }}(0)}{r_{\text {bound }}(100)}, 2: \frac{d_{\mathrm{EM}}(0)}{d_{\mathrm{EM}}(100)}, 3: \frac{d_{\mathrm{mem}}(0)}{d_{\mathrm{mem}}(100)}, 4: \frac{n(0)}{n(100)}$, 5: $d_{\mathrm{EM}}(0), 6: \frac{d_{\mathrm{EM}}(0)}{d_{\mathrm{mem}}(0)}, 7: \frac{d_{\mathrm{EM}}(100)}{d_{\mathrm{mem}}(100)}$. Ranges are given in section A.6. Scales are logarithmic except for $D_{\mathrm{EM}}^{m}, D_{n}$, and $\frac{r_{\text {bound }}(0)}{r_{\text {bound }}(100)}$. 


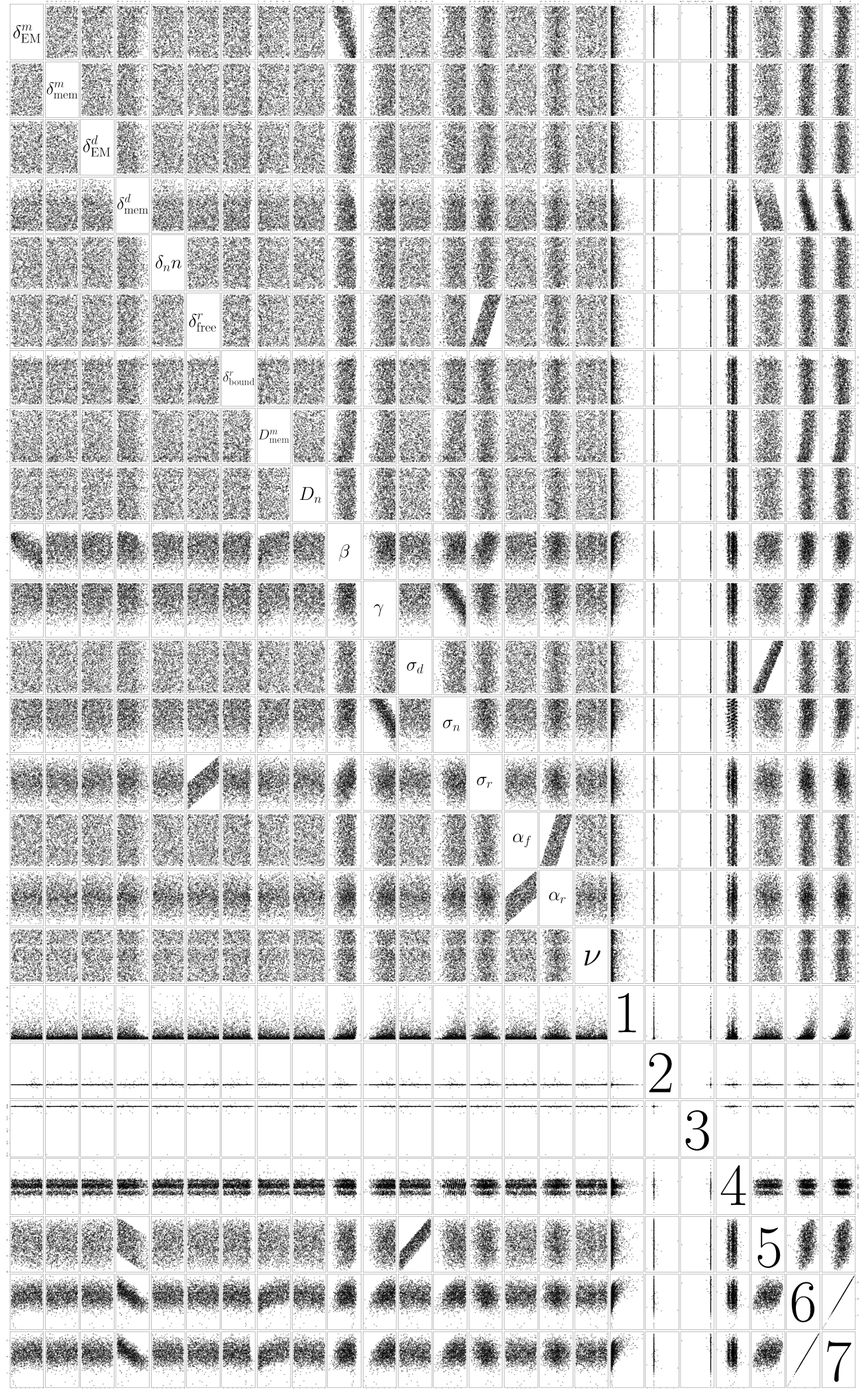

Figure 6: Pairwise plot of parameters for which the model with global Notum synthesis gives rise to a gradient of bound receptor meeting the conditions set out in section 3.1. Number correspondences are the same as in Figure 5. 
wide variety of kinetic parameters. An example gradient is shown in Figure 2 .

For $4.4 \%$ of the parameters tested, the concentration of bound receptor did not form a proper gradient, in that the concentration did not steadily decrease as the distance from the source of the morphogen increased. This is illustrated in Figure 3. Figure 4 shows the concentrations of other variables of the same system; the increasing concentration of morphogen is associated with an increasing membrane Dll/Dlp concentration (and also an increasing ratio of membrane to EM Dll/Dlp, as the latter is constant).

To examine the influence of the parameters on the establishment of the desired gradient, satisfactory parameters were plotted pairwise, along with measures of the gradients established (Figure 5). It resulted from this analysis that for all satisfactory bound-receptor gradients, the gradient of Dll/Dlp was extremely shallow (with no more than $3 \%$ variation across the field for the EM form, with an average of $0.16 \%$, and no more than $29 \%$ variation for the membrane form, with an average of $3 \%$ ), as was that of Notum (with a maximum variation of $10 \%$ and an average of $3 \%$ ). Diffusion coefficients for Notum were biased towards higher values, and correlated strongly with the amplitude of both Notum and Dll/Dlp gradients.

This suggested that localized production of Notum was not favorable to the establishment of bound receptor gradient.

\subsection{Glycoprotein phase-repartition with global Notum}

In order to test this, the same simulation was run, but with Notum synthesis over the whole field, rather than over the first $10 \mu \mathrm{m}$. Pairwise plots of satisfactory parameters are shown in Figure 6. About $1.7 \%$ of the parameter tested (out of about 90,000 tested) met the conditions, i.e. 3 -fold more than with localized Notum synthesis, with ranges of up to 30-fold (range average was 4.6 ). Only in $0.9 \%$ of the cases was the gradient reversed as in Figure 3.

Comparison of Figures 5 and 6 shows that global Notum synthesis relaxes restriction on the relative values of the morphogen diffusion rate $D^{m}$ and $\beta$, the rate of exchange of the morphogen between the membrane and EM phases (the former must be sufficiently high compared to the latter, if Notum is synthesized locally), as well as the restriction on the relative values of $D^{m}$ and the activity of Notum $\gamma$ (again, the former tends to be high compared to the latter, if Notum is synthesized locally).

Notable restrictions on parameter sets common to the models with localized or global Notum synthesis are that the product of $\delta_{E M}^{m}$, the degradation rate of the morphogen in the extracellular matrix and $\beta$, as well as that of the activity of Notum $\gamma$ and its synthesis rate $\sigma_{n}$, must be sufficiently high. 
Interestingly, the rate of association of receptor and morphogen is not skewed in the $10^{5}-10^{8}$ range; the proposed model thus does not share the restriction of that of Lander et al. (2002). Also, to investigate the role of bound-receptor downregulation, the corresponding parameter $\delta_{\text {bound }}^{r}$ was set to 0 , and the same analysis as previously carried out. The results were roughly identical (data not shown), showing that receptor downregulation is not an important feature of this model.

\subsection{Shh-like oligomerization}

Different simulations were run, varying the number of oligomer forms considered (from 3 to 38), the form under which Shh was injected (as a monomer or a multimer), and the rate constants for the oligomerization reaction $x_{i}+x_{j} \leftrightarrow$ $x_{i+j}$, where $x_{p}$ is a p-mer of Shh. The laws tried out for the forward rate constants were $k_{i, j}^{+}=k^{+}$(i.e. the same rate for all reactions), $k_{i, j}^{+}=k^{+} /(i+j)$ (i.e. "mild" scaling down of the rates according to the size of the product), and $k_{i, j}^{+}=k^{+} /(i+j)^{2}$ (stronger scaling down of the rates). Backward reactions were considered to happen all at the same rate.

No suitable gradients were identified with 18-mer Shh injection at the boundary (rather than monomer Shh), or with strong scaling of the oligomerization rates. It was easiest to identify suitable parameter sets for a system with Shh forming up to 10-mers, with no scaling of forward reaction rates, and for systems with Shh forming up to 8-mers or 18-mers, with mild scaling of forward reaction rates.

The low number of parameter sets identified (about 60) does not warrant a detailed parameter study. An example gradient is shown in Figure 7.

\section{Discussion}

We have detailed two mechanisms for the creation of a morphogen gradient, which do not share restrictions of mechanisms which have been previously studied:

- Separation of the morphogen into an extracellular phase, from which it cannot directly interact with receptors, and a cell-membrane phase; the corresponding mathematical model does not require any degradation scheme of receptor-morphogen complexes, and can accommodate biochemical parameters over a very wide range of values. Values which show correlation when interesting gradients are formed are the relative rates of morphogen degradation and phase equilibration, as well 

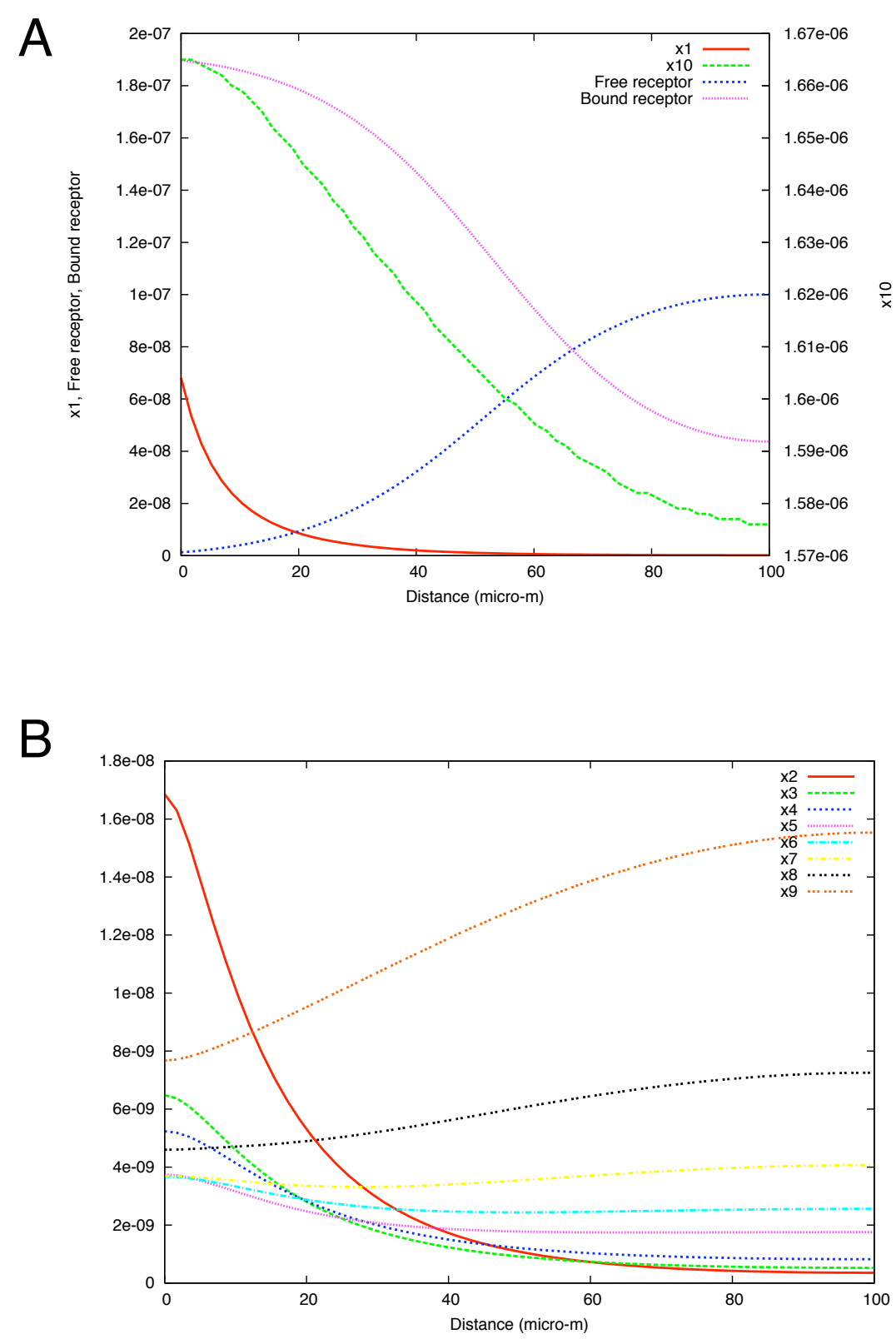

Figure 7: Example of a suitable gradient of bound receptor for the Shh-like oligomerization model, with formation of up to 10-mers and no scaling of forward reaction rates, after $15625 \mathrm{~s}$ of simulation. (A) Concentrations of the monomer $x_{1}$, the maximal-size multimer $x_{10}$, and free and bound receptors; note the different scale for $x_{10}$ (B) Concentrations of intermediary multimer forms. 
as rates related to the relative levels of extracellular matrix and cellmembrane concentrations. These are good candidates for experimental observation and manipulation.

- Oligomerization of the morphogen into a compound which does not interact with receptors, as could be the case for Sonic Hedgehog.

Both these mechanisms create in effect a dynamic sub-population of morphogen that travels unhindered by receptor interaction, allowing the morphogen gradient not to die off too quickly or saturate receptors. Tracking of the membrane diffusion of single molecules is feasible (as shown for example by Murakoshi et al., 2004); extending these experiments to extracellular diffusion would allow one to assess whether sets of individual paths indeed reflect the sort of diffusion which underlies these new models.

The phase repartition model is compatible with both observations that proteoglycans are indispensable for Dpp and Wg diffusion, and that cell membrane-tethered diffusion would be too slow to account for the observed speed of gradient establishment (Lander et al., 2002). Its absence of requirement for receptor-mediated morphogen degradation is also in line with the lack of requirement of the receptors $\mathrm{Fz}$ and Fz2 to establish a Wg gradient (Han et al., 2005).

It has only recently been discovered that Dlp's GPI anchor can be cleaved, releasing it into the extracellular matrix. Since Notum is synthesized only in a region of high $\mathrm{Wg}$ signaling, it has been proposed that this localized expression has a role in promoting morphogen diffusion to regions of less intense signaling. However, our model makes the counter-intuitive suggestion that this may not be the case. This is in line with experiments showing that misexpression of Notum in the dorsal compartment of a Drosophila wing disc has symmetrical effects on the dorsal and ventral compartments (Giráldez et al., 2002), and with the fact that Dpp has been show to also require Dll and Dlp for its diffusion (Belenkaya et al., 2004). If Notum (and the EM and membrane fractions of Dll and Dlp) had a strongly non-homogenous distribution along the dorso-ventral axis, one would expect interference with Dpp signalling, which takes place in a gradient orthogonal to that of $\mathrm{Wg}$ (Figure 1).

Semi-quantitative imaging of the membrane-associated and free, extracellular sub-populations would provide crucial data to test models with. In particular, to accomodate experimental data showing that morphogen diffusion does not occur over clones deficient in Dll and Dlp (Belenkaya et al., 2004, Han et al., 2005), the phase repartition model makes the important assumption that extracellular Dlp has a negligible diffusion rate. 
Even though parameter sets were identified which allow Shh-like oligomerization to give rise to suitable gradients, this did not happen as readily as for the phase repartition model. This could very well have to do with the fact that glycoproteins also seem to be essential to the establishment of a Shh gradient (Han et al., 2004), and that the two phenomena need to be taken into account together.

\section{Acknowledgements}

This work was funded by an AstraZeneca scholarship awarded to OC by CoMPLEX. I am grateful to Amanda Albazerchi and Christian Bottomley for discussions, and to Daniel Brewer for computational resources.

\section{References}

Baeg G., Lin X., Khare N., Baumgartner S., and Perrimon N. Heparan sulfate proteoglycans are critical for the organization of the extracellular distribution of wingless. Development, 128(1):87-94, 2001. Abstract. 2.1

Baeg G., Selva E., Goodman R., Dasgupta R., and Perrimon N. The wingless morphogen gradient is established by the cooperative action of frizzled and heparan sulfate proteoglycan receptors. Dev Biol, 276(1):89-100, 2004. Abstract. Full text. 2

Belenkaya T., Han C., Yan D., Opoka R., Khodoun M., Liu H., and Lin X. Drosophila dpp morphogen movement is independent of dynamin-mediated endocytosis but regulated by the glypican members of heparan sulfate proteoglycans. Cell, 119(2):231-44, 2004. Abstract. Full text. 2, 2.1, 4

Briscoe J., Chen Y., Jessell T., and Struhl G. A hedgehog-insensitive form of patched provides evidence for direct long-range morphogen activity of sonic hedgehog in the neural tube. Mol Cell, 7(6):1279-91, 2001. Abstract. 2.1

Cadigan K. Regulating morphogen gradients in the drosophila wing. Semin Cell Dev Biol, 13(2):83-90, 2002. Abstract. 2

Chen M., Li Y., Kawakami T., Xu S., and Chuang P. Palmitoylation is required for the production of a soluble multimeric hedgehog protein complex and long-range signaling in vertebrates. Genes Dev, 18(6):641-59, 2004. Abstract. Full text. 2.2 
Doctor J., Jackson P., Rashka K., Visalli M., and Hoffmann F. Sequence, biochemical characterization, and developmental expression of a new member of the tgf-beta superfamily in drosophila melanogaster. Dev Biol, 151 (2):491-505, 1992. Abstract. A.1

Dyson S. and Gurdon J. The interpretation of position in a morphogen gradient as revealed by occupancy of activin receptors. Cell, 93(4):557-68, 1998. Abstract. A.1

Eldar A., Rosin D., Shilo B., and Barkai N. Self-enhanced ligand degradation underlies robustness of morphogen gradients. Dev Cell, 5(4):635-46, 2003. Abstract. 2, A.2

Feng J., White B., Tyurina O., Guner B., Larson T., Lee H., Karlstrom R., and Kohtz J. Synergistic and antagonistic roles of the sonic hedgehog nand c-terminal lipids. Development, 131(17):4357-70, 2004. Abstract. Full text. 2.2

Freeman M. and Gurdon J. Regulatory principles of developmental signaling. Annu Rev Cell Dev Biol, 18:515-39, 2002. Abstract. Full text. A.1

Gabdoulline R. and Wade R. Simulation of the diffusional association of barnase and barstar. Biophys J, 72(5):1917-29, 1997. Abstract. A.1

Gerlitz O. and Basler K. Wingful, an extracellular feedback inhibitor of wingless. Genes Dev, 16(9):1055-9, 2002. Abstract. Full text. 2.1

Giráldez A., Copley R., and Cohen S. Hspg modification by the secreted enzyme notum shapes the wingless morphogen gradient. Dev Cell, 2(5): 667-76, 2002. Abstract. 2.1, 4

Grimm S. and Pflugfelder G. Control of the gene optomotor-blind in drosophila wing development by decapentaplegic and wingless. Science, 271(5255):1601-4, 1996. Abstract. A.2

Gurdon J., Standley H., Dyson S., Butler K., Langon T., Ryan K., Stennard F., Shimizu K., and Zorn A. Single cells can sense their position in a morphogen gradient. Development, 126(23):5309-17, 1999. Abstract. A.1

Han C., Belenkaya T., Wang B., and Lin X. Drosophila glypicans control the cell-to-cell movement of hedgehog by a dynamin-independent process. Development, 131(3):601-11, 2004. Abstract. Full text. 2, 4 
Han C., Yan D., Belenkaya T., and Lin X. Drosophila glypicans dally and dally-like shape the extracellular wingless morphogen gradient in the wing disc. Development, 132(4):667-79, 2005. Abstract. Full text. 2, 2.1, 4

Kerszberg M. and Wolpert L. Mechanisms for positional signalling by morphogen transport: a theoretical study. J Theor Biol, 191(1):103-14, 1998. Abstract. 2, A.1

Khare N. and Baumgartner S. Dally-like protein, a new drosophila glypican with expression overlapping with wingless. Mech Dev, 99(1-2):199-202, 2000. Abstract. 2.1

Koenig B., Cook J., Wolsing D., Ting J., Tiesman J., Correa P., Olson C., Pecquet A., Ventura F., and Grant R. Characterization and cloning of a receptor for bmp-2 and bmp-4 from nih 3t3 cells. Mol Cell Biol, 14(9): 5961-74, 1994. Abstract. A.1

Kreuger J., Perez L., Giraldez A., and Cohen S. Opposing activities of dallylike glypican at high and low levels of wingless morphogen activity. Dev Cell, 7(4):503-12, 2004. Abstract. Full text. 2.1

Kruse K., Pantazis P., Bollenbach T., Jülicher F., and González-Gaitán M. Dpp gradient formation by dynamin-dependent endocytosis: receptor trafficking and the diffusion model. Development, 131(19):4843-56, 2004. Abstract. Full text. 2, A.1

Lander A., Nie Q., and Wan F. Do morphogen gradients arise by diffusion? Dev Cell, 2(6):785-96, 2002. Abstract. 2, 3, 3.2, 4, A.1, A.2

Murakoshi H., Iino R., Kobayashi T., Fujiwara T., Ohshima C., Yoshimura A., and Kusumi A. Single-molecule imaging analysis of ras activation in living cells. Proc Natl Acad Sci U S A, 101(19):7317-22, 2004. Abstract. Full text. 4

Nellen D., Burke R., Struhl G., and Basler K. Direct and long-range action of a dpp morphogen gradient. Cell, 85(3):357-68, 1996. Abstract. 2.1

Northrup S. and Erickson H. Kinetics of protein-protein association explained by brownian dynamics computer simulation. Proc Natl Acad Sci USA, 89(8):3338-42, 1992. Abstract. A.1

Nugent M. and Edelman E. Kinetics of basic fibroblast growth factor binding to its receptor and heparan sulfate proteoglycan: a mechanism for cooperactivity. Biochemistry, 31(37):8876-83, 1992. Abstract. A.1 
Shimmi O. and O'Connor M. Physical properties of tld, sog, tsg and dpp protein interactions are predicted to help create a sharp boundary in bmp signals during dorsoventral patterning of the drosophila embryo. Development, 130(19):4673-82, 2003. Abstract. Full text. A.1

Staehling-Hampton K., Jackson P., Clark M., Brand A., and Hoffmann F. Specificity of bone morphogenetic protein-related factors: cell fate and gene expression changes in drosophila embryos induced by decapentaplegic but not 60a. Cell Growth Differ, 5(6):585-93, 1994. Abstract. A.2

Suzuki A., Thies R., Yamaji N., Song J., Wozney J., Murakami K., and Ueno N. A truncated bone morphogenetic protein receptor affects dorsal-ventral patterning in the early xenopus embryo. Proc Natl Acad Sci U S A, 91 (22):10255-9, 1994. Abstract. A.1

Tao L. and Nicholson C. Diffusion of albumins in rat cortical slices and relevance to volume transmission. Neuroscience, 75(3):839-47, 1996. Abstract. A.1

Tsuda M., Kamimura K., Nakato H., Archer M., Staatz W., Fox B., Humphrey M., Olson S., Futch T., Kaluza V., Siegfried E., Stam L., and Selleck S. The cell-surface proteoglycan dally regulates wingless signalling in drosophila. Nature, 400(6741):276-80, 1999. Abstract. Full text. 2.1

Zecca M., Basler K., and Struhl G. Direct and long-range action of a wingless morphogen gradient. Cell, 87(5):833-44, 1996. Abstract. 2.1, A.2

Zeng X., Goetz J., Suber L., Scott W., Schreiner C., and Robbins D. A freely diffusible form of sonic hedgehog mediates long-range signalling. Nature, 411(6838):716-20, 2001. Abstract. Full text. 2.2

\section{A Appendix}

\section{A.1 Parameter values}

The concentrations of morphogens and receptors, as well as their kinetic reaction coefficients, have been precisely evaluated only in a restricted number of cases (for example by Dyson and Gurdon, 1998; see Freeman and Gurdon, 2002 for a review). Relevant concentration ranges for morphogens have been proposed to be around 10-100 pM (Freeman and Gurdon, 2002). Dyson and Gurdon (1998) have shown that cells can respond with as few as $2 \%$ 


\begin{tabular}{|r|r|r|}
\hline Parameters & Range & Distribution \\
\hline$\delta_{\mathrm{EM}}^{m}, \delta_{\mathrm{mem}}^{m}, \delta_{\mathrm{EM}}^{d}, \delta_{\mathrm{mem}}^{d}, \delta_{n}, \delta_{\text {free }}^{r}, \delta_{\text {bound }}^{r}, \delta_{i}$ & $10^{-7}-10^{-4} s^{-1}$ & Log-uniform \\
$D_{\mathrm{EM}}^{m}, D^{n}, D_{i}$ & $5-30 \mu m^{2} \cdot s^{-1}$ & Uniform \\
$\beta$ & $10^{-8}-1 s^{-1}$ & Log-uniform \\
$\gamma$ & $1-10^{6} s^{-1} M^{-1}$ & Log-uniform \\
$\sigma_{d}, \sigma_{n}$ & $10^{-8}-10^{-13} M s^{-1}$ & Log-uniform \\
$\sigma_{r}$ & $10^{-14}-10^{-8} M s^{-1}$ & $\frac{\sigma_{r}}{\delta_{\text {free }}^{r}}$ log-uniform in $10^{-7}-10^{-4}$ \\
$\alpha_{f}, k^{+}$ & $10^{5}-10^{8} M^{-1} s^{-1}$ & Log-uniform \\
$\alpha_{r}, k^{-}$ & $10^{-7}-10^{-1} s^{-1}$ & $\frac{\alpha_{f}}{\alpha_{r}}, \frac{k^{+}}{k^{-}}$log-uniform in $10^{5}-10^{8}$ \\
$\nu$ & $10^{-8}-10^{-13} M \mu m^{-1}$ & Log-uniform \\
\hline
\end{tabular}

Table 1: Ranges from which parameters were selected at random for the simulations described in section 3 .

of their receptors bound to the morphogen (the total of number of receptors per cell being estimated to 5,000); this gives a maximum 50-fold useful variation range of the concentration of bound receptor (also compatible with the data of Gurdon et al., 1999). Cultured S2 cells show responses to Dpp over a 10-fold range, from 100pM to 1000pM (Shimmi and O'Connor, 2003) (but the presence of Dlp could possibly alter those responses). BMP receptor affinity for its ligand is of the order of nanomolars (Koenig et al., 1994, Suzuki et al., 1994), and that of the FGF receptor picomolars (Nugent and Edelman, 1992).

The association rates of two proteins in solution can be as high as $10^{8}$ $10^{9} \mathrm{M}^{-1} \mathrm{~s}^{-1}$, close to the rate of random encounter given by Smoluchowski's equation $k_{\mathrm{on}}=4 \Pi D R$, probably thanks to long-range electrostatic interactions (Northrup and Erickson, 1992, Gabdoulline and Wade, 1997). The order of magnitude of binding rates seems however to normally be around $10^{6} M^{-1} s^{-1}$. It has been proposed that, for BMP-like morphogens, it can be as low as $3.10^{5} M^{-1} s^{-1}$ (references in Lander et al., 2002). For FGF, the binding rate has been measured at $4.10^{6} M^{-1} s^{-1}$ (Nugent and Edelman, 1992); such high values have been used in some simulations (Kruse et al., 2004).

The extracellular diffusion rate of a $50-\mathrm{kDa}$ albumin, in vivo, has been reported to be $16 \mu \mathrm{m}^{2} \mathrm{~s}^{-1}$, and that of a $15-\mathrm{kDa}$ albumin $24 \mu \mathrm{m}^{2} \mathrm{~s}^{-1}$ (Tao and Nicholson, 1996). The molecular weights of processed Wingless and Decapentaplegic dimers are about 36kDa (value from Pubmed protein) and $30 \mathrm{kDa}$ (Doctor et al., 1992), respectively. $10 \mu \mathrm{m}^{2} s^{-1}$ seems therefore to be a good assumption for the order of magnitude of the extracellular diffusion rates (this is the value used by Kerszberg and Wolpert, 1998, and Lander et al., 2002). 


\section{A.2 Evaluation of gradients}

Small dependence of the gradient on the rate of morphogen production has been used as a criterion of biological relevance (Eldar et al., 2003). There are however documented cases of morphogen readouts not being buffered against changes in morphogen production rates (Grimm and Pflugfelder, 1996, Staehling-Hampton et al., 1994, Zecca et al., 1996). In this study, gradients were evaluated with the " $\eta$ " criterion proposed by Lander et al. (2002), which provides a simple measure of how close to linearity they are (as it would be difficult for rapidly-decaying gradients to provide a useful range of concentrations over a high number of cells).

\section{A.3 Simulations}

Simulations were performed with the Numerical Algorithms Group's Fortran library Mark 20, using the D03PDF function, called from a custom Fortran 77 program (available on request), compiled with the NAGWare compiler, and run on G5 iMacs and PowerMacs. The D03PDF accuracy setting was $10^{-12}$, and the degree of the polynomial approximation 2 or 3 .

\section{A.4 Equations for Wg-like diffusion}

$$
\begin{aligned}
& \dot{m}_{\mathrm{EM}}=D_{\mathrm{EM}}^{m} \Delta m_{\mathrm{EM}}-\delta_{\mathrm{EM}}^{m} m_{\mathrm{EM}}+\beta \frac{m_{\mathrm{mem}} d_{\mathrm{EM}}-m_{\mathrm{EM}} d_{\mathrm{mem}}}{d_{\mathrm{EM}}+d_{\mathrm{mem}}} \\
& \dot{m}_{\mathrm{mem}}=\quad-\delta_{\mathrm{mem}}^{m} m_{\mathrm{mem}}-\beta \frac{m_{\mathrm{mem}} d_{\mathrm{EM}}-m_{\mathrm{EM}} d_{\mathrm{mem}}}{d_{\mathrm{EM}}+d_{\mathrm{mem}}} \\
& -\alpha_{f} r_{\text {free }} m_{\text {mem }}+\alpha_{r} r_{\text {bound }} \\
& \dot{d}_{\mathrm{EM}}=\quad-\delta_{\mathrm{EM}}^{d} d_{\mathrm{EM}}+\gamma n d_{\mathrm{mem}} \\
& \dot{d}_{\text {mem }}=\quad-\delta_{\text {mem }}^{d} d_{\text {mem }}-\gamma n d_{\text {mem }}+\sigma_{d} \\
& \dot{n}=D_{n} \Delta n \quad-\delta_{n} n+\sigma_{n} \\
& \dot{r}_{\text {free }}=\quad-\delta_{\text {free }}^{r} r_{\text {free }}-\alpha_{f} r_{\text {free }} m_{\text {mem }}+\alpha_{r} r_{\text {bound }}+\sigma_{r} \\
& \dot{r}_{\text {bound }}=\quad-\delta_{\text {bound }}^{r} r_{\text {bound }}+\alpha_{f} r_{\text {free }} m_{\text {mem }}-\alpha_{r} r_{\text {bound }} \\
& \frac{\partial m_{\mathrm{EM}}}{\partial x}(x=0)=\quad-\nu \\
& d_{\text {mem }}(t=0)=\quad \sigma_{d} / \delta_{\text {mem }}^{d} \\
& r_{\text {free }}(t=0)=\quad \sigma_{r} / \delta_{\text {free }}^{r}
\end{aligned}
$$


Unspecified boundary conditions are zero-flux conditions, and unspecified initial conditions are 0 .

\section{A.5 Equations for Shh-like oligomerization}

$$
\begin{aligned}
\dot{x}_{1}= & D_{1} \Delta x_{1}-\delta_{1} x_{1}+\Sigma_{j>1} k_{1, j-1}^{-} x_{j}+k_{1,1}^{-} x_{2}-\Sigma_{j=1 . . n-1} k_{1, j}^{+} x_{1} x_{j} \\
& -\alpha_{f} r_{\text {free }} x_{1}+\alpha_{r} r_{\text {bound }} \\
\dot{x}_{i, 1<i \leq n}= & D_{i} \Delta x_{i}-\delta_{i} x_{i}+\Sigma_{j+k=i, j>k}\left(k_{j, k}^{+} x_{j} x_{k}-k_{j, k}^{-} x_{i}\right) \\
& +\Sigma_{j>i} k_{i, j-i}^{-} x_{j}+k_{i, i}^{-} x_{2 i}-\Sigma_{j=1 . . n-i} k_{i, j}^{+} x_{i} x_{j}-1_{2 i \leq n} k_{i, i}^{+} x_{i}^{2} \\
\dot{r}_{\text {free }}= & -\delta_{\text {free }}^{r} r_{\text {free }}-\alpha_{f} r_{\text {free }} x_{1}+\alpha_{r} r_{\text {bound }}+\sigma_{r} \\
\dot{r}_{\text {bound }}= & -\delta_{\text {bound }}^{r} r_{\text {bound }}+\alpha_{f} r_{\text {free }} x_{1}-\alpha_{r} r_{\text {bound }} \\
\frac{\partial x_{1}}{\partial x}(x=0)= & -\nu \\
r_{\text {free }}(t=0)= & \sigma_{r} / \delta_{\text {free }}^{r}
\end{aligned}
$$

where $x_{i}$ is an i-mer of Shh, $n$ is the maximum number of Shh proteins which can associate into a single oligomer, $k_{i, j}^{+}$and $k_{i, j}^{-}$are respectively the association and dissociation rates in the reaction $x_{i}+x_{j} \leftrightarrow x_{i+j}$, and the other parameters are the same as previously. Unspecified boundary conditions are zero-flux conditions, and unspecified initial conditions are 0.

The diffusion rates for oligomers were scaled from the rate for the monomer, according to the Stokes-Einstein law for spherical particles: $D_{i}=D_{1} * i^{-1 / 3}$. 


\section{A.6 Ranges for the measures plotted in figures 5 and 6}

Figure 5:

\begin{tabular}{|r|r|r|r|}
\hline Number & Measure & Lower bound & Upper bound \\
\hline 1 & $\frac{r_{\text {bound }}(0)}{r_{\text {bound }}(100)}$ & 2 & 34 \\
2 & $\frac{d_{\mathrm{EM}}(0)}{d_{\mathrm{EM}}(100)}$ & $-2.9 .10^{-2}$ & $6.1 .10^{-3}$ \\
3 & $\frac{d_{\operatorname{mem}}(0)}{d_{\operatorname{mem}(100)}}$ & -0.34 & -0.015 \\
4 & $\frac{n(0)}{n(100)}$ & 0.015 & 0.084 \\
5 & $d_{\mathrm{EM}}(0)$ & -19 & -2.9 \\
6 & $\frac{d_{\mathrm{EM}}(0)}{d_{\operatorname{mem}}(0)}$ & 3.0 & 17.7 \\
7 & $\frac{d_{\mathrm{EM}(100)}}{d_{\operatorname{mem}}(100)}$ & 2.9 & 17.7 \\
\hline
\end{tabular}

Figure 6:

\begin{tabular}{|r|r|r|r|}
\hline Number & Measure & Lower bound & Upper bound \\
\hline 1 & $\frac{r_{\text {bound }}(0)}{r_{\text {bound }}(100)}$ & 2 & 30 \\
2 & $\frac{d_{\mathrm{EM}}(0)}{d_{\mathrm{EM}}(100)}$ & $-1.1 .10^{-12}$ & $3.6 .10^{-12}$ \\
3 & $\frac{d_{\operatorname{mem}}(10)}{d_{\mathrm{mem}(100)}}$ & $-4.10^{-11}$ & $3.10^{-12}$ \\
4 & $\frac{n(0)}{n(100)}$ & $-8.10^{-15}$ & $1.9 .10^{-14}$ \\
5 & $d_{\mathrm{EM}(0)}$ & -19 & -3.0 \\
6 & $\frac{d_{\mathrm{EM}}(0)}{d_{\mathrm{mem}}(0)}$ & 1.3 & 17 \\
7 & $\frac{d_{\mathrm{EM}}(100)}{d_{\mathrm{mem}}(100)}$ & 1.3 & 17 \\
\hline
\end{tabular}

